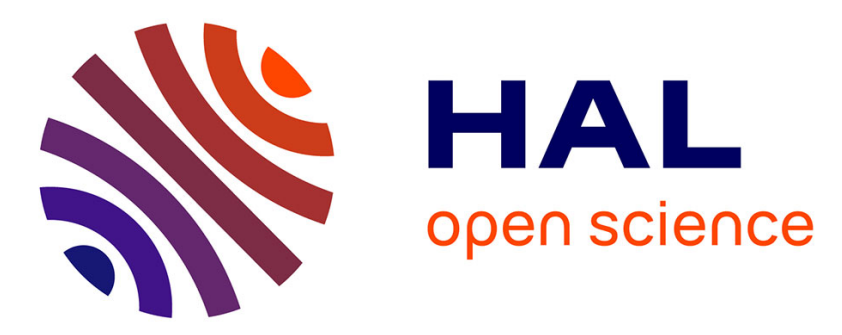

\title{
A stronger necessary condition for the multistationarity of chemical reaction networks
}

Sylvain Soliman

\section{To cite this version:}

Sylvain Soliman. A stronger necessary condition for the multistationarity of chemical reaction networks. Bulletin of Mathematical Biology, 2013, 75 (11), pp.2289-2303. 10.1007/s11538-013-9893-7 . hal-00772438v5

\author{
HAL Id: hal-00772438 \\ https://hal.inria.fr/hal-00772438v5
}

Submitted on 16 May 2013

HAL is a multi-disciplinary open access archive for the deposit and dissemination of scientific research documents, whether they are published or not. The documents may come from teaching and research institutions in France or abroad, or from public or private research centers.
L'archive ouverte pluridisciplinaire HAL, est destinée au dépôt et à la diffusion de documents scientifiques de niveau recherche, publiés ou non, émanant des établissements d'enseignement et de recherche français ou étrangers, des laboratoires publics ou privés. 


\title{
A stronger necessary condition for the multistationarity of chemical reaction networks
}

\author{
Sylvain Soliman
}

the date of receipt and acceptance should be inserted later

\begin{abstract}
Biochemical reaction networks grow bigger and bigger, fed by the high-throughput data provided by biologists and bred in open repositories of models allowing merging and evolution. Nevertheless, since the available data is still very far from permitting the identification of the increasing number of kinetic parameters of such models, the necessity of structural analyses for describing the dynamics of chemical networks appears stronger every day.

Using the structural information, notably from the stoichiometric matrix, of a biochemical reaction system, we state a more strict version of the famous Thomas' necessary condition for multistationarity. In particular, the obvious cases where Thomas' condition was trivially satisfied, mutual inhibition due to a multimolecular reaction and mutual activation due to a reversible reaction, can now easily be ruled out.

This more strict condition shall not be seen as some version of Thomas' circuit functionality for the continuous case but rather as related and complementary to the whole domain of the structural analysis of (bio)chemical reaction systems, as pioneered by the Chemical Reaction Network Theory.
\end{abstract}

Keywords Jacobian matrix · Influence graph · Feedback circuit · Multistationarity · Chemical reaction network

Mathematics Subject Classification (2000) 92C42 - 34C10 - 34C23 .

\section{Introduction}

In the last thirty years, the conjecture of Thomas [25] on the necessary presence of a positive circuit for the occurrence of multistationarity has opened a whole field of research.

First on the mathematical side, since it has been proven under various forms, depending on the restrictions on the system: discrete $[20,19]$ or continuous $[18,13,22,5,24]$.

But also based on the insight gained from it allowing better modeling and understanding of biochemical networks, especially in the emerging field of Systems Biology.

Sylvain Soliman

Inria Paris-Rocquencourt - Equipe CONTRAINTES

Domaine de Voluceau, Rocquencourt, BP 105

78153 LE CHESNAY CEDEX - FRANCE

E-mail: Sylvain.Soliman@inria.fr 
However, if that second aspect is striking in the field of discrete modeling of gene regulatory networks, even the stronger versions of Thomas' necessary conditions published recently [16] did not have the same impact in the Ordinary Differential Equations (ODE) based modeling community.

This is mostly due to the fact that this necessary condition, the existence of a positive loop in the Jacobian of the ODE system, is almost always satisfied. Indeed, any binary reaction equipped with Mass-Action kinetics will lead to the mutual inhibition of the two substrates, and thus create such a loop (see Fig. 1a).

Even a reversible unary reaction can lead to satisfying the condition, since it induces a mutual activation of the two biochemical species (see Fig. 1b).

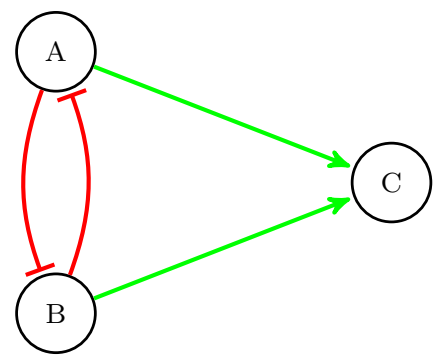

(a)

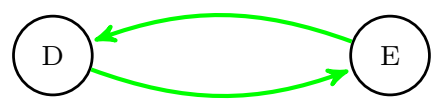

(b)

Fig. 1 (a): mutual inhibition resulting from $A+B \Rightarrow$ C. (b): mutual activation resulting from $D \Leftrightarrow$ E. For clarity, negative self-loops have not been represented

Since many models are still being constructed based on ODEs, the community turned to other types of conditions, especially for purely Mass-Action or Non Auto-Catalytic (NAC) systems [8, $6]$.

Nevertheless, even using these conditions, [7] states that enzyme kinetics of the form: $\mathrm{S}+\mathrm{E} \Leftrightarrow \mathrm{C} \Rightarrow \mathrm{P}+\mathrm{E}$ promote cycles and thus might explain why such systems might be far more prone than others to exhibit multiple equilibria.

In this article, after some preliminaries, we give necessary conditions for multistationarity, not restricted to Mass-Action or NAC chemical reaction systems, stronger than Thomas', and local as in [24]. They show that enzyme kinetics of the above form-Fig. 1 or enzyme kinetics à la [7]do not by themselves create multistationarity-inducing cycles. This is shown through a main theorem and three practical corollaries, and is extended in Section 5 via graph transformation operations. We then position these new results with respect to existing works and conclude on possible perspectives.

Our approach is based on the Directed Species Reaction (DSR) graph as defined in [15] by Kaltenbach (and not the homonym from [3]) and the related analysis of terms appearing in the Jacobian's determinant. It is local, i.e., the existence of a positive loop can be verified for a given point of the phase space. Note that since the local version of Thomas' conjecture for oscillations has been proven false in [21], there is no direct generalization of our work to the existence of limit cycles, even using Hurwitz determinants as analogous to principal minors for Hopf bifurcations. 


\section{Jacobian characterization of multistationarity}

Following Soulé [24] we will use the Gale-Nikaido univalence theorem [12] and its refinements.

We consider a differentiable map $F$ from $\Omega$, a product of $n$ intervals of $\mathbb{R}$, to $\mathbb{R}^{n}$ and study the corresponding system $\dot{x}=F(x)$. Its Jacobian matrix, noted $J$, is defined as usual as follows:

$$
J_{i j}(x)=\partial f_{i} / \partial x_{j}(x)
$$

The influence graph associated to $J$ at each point $x$ is the labelled directed graph with vertices $\left\{x_{i} \mid 1 \leq i \leq n\right\} ;$ an arc $\left(x_{i}, x_{j}\right)$ if $J_{i j}$ is not null, and the sign of $J_{i j}$ as label.

The graphs depicted in Fig. 1 are influence graphs where the labels are represented as colors and arrow tips: green and pointy tip for a positive sign, red and T-tip for a negative sign.

From now on we shall use the following terms: a Hooping of $J$ is a disjoint collection of cycles of its influence graph; it is Hamiltonian if it is covering all $n$ vertices.

\section{Theorem 1 ([24])}

Let $F$ be any differentiable map from $\Omega$ to $\mathbb{R}^{n}$, with Jacobian matrix $J$. If $\Omega$ is open and $F$ has two nondegenerate zeroes in $\Omega$ then there exists a in $\Omega$ such that some principal minor of $-J(a)$ is negative.

Using the Leibniz formula for determinants that defines them in terms of permutations of the indices of the matrix, and the link between those and hamiltonian hoopings, one then obtains the classical necessary condition on the existence of some $a$ such that there is a positive circuit in $J(a)$.

Note that this result can be extended to the case where $\Omega$ is the (closed) positive orthant, when some additional, but commonly accepted in the Systems Biology community, conditions are met. This is the case for instance if $F$ is the function associated with the dynamics of a chemical reaction system with only Mass-Action kinetics [6], or if one is only interested in multistationarity associated to Saddle-node bifurcations.

\section{Directed Species-Reaction graph}

Let us now consider a (bio)chemical reaction system with $n$ species $S_{1}, \ldots, S_{n}$ and $m$ reactions $R_{1}, \ldots, R_{m}$. Using notations from [15] we write:

$$
R_{j}=\sum_{i=1}^{n} y_{i j} S_{i} \longrightarrow \sum_{i=1}^{n} y_{i j}^{\prime} S_{i}
$$

The $y$ and $y^{\prime}$ represent the stoichiometric coefficients of the reactants and products of the reaction.

The rate law associated with reaction $R_{j}$ will be written $v_{j}$. This defines a dynamical system in the sense of previous section: $\dot{x}=F(x)$ where $x_{i}$ is the concentration of species $S_{i}$ and

$$
f_{i}(x)=\sum_{j} v_{j}(x) \cdot\left(y_{i j}^{\prime}-y_{i j}\right)
$$

This kind of systems encompasses most of the Systems Biology models developed nowadays. One can notice for instance that the Systems Biology Markup Language (SBML) [14] can be translated to such reactions by splitting reversible reactions into forward and backward reactions and by including modifiers on both sides of the reaction (they are not affected by it, but do affect it). 
Such a system can be represented naturally in a graphical form as a bipartite graph for species and reactions, as depicted in Fig. 2. Arcs can also be labelled with the $y$ and $y^{\prime}$ in a Petri-net-like manner.

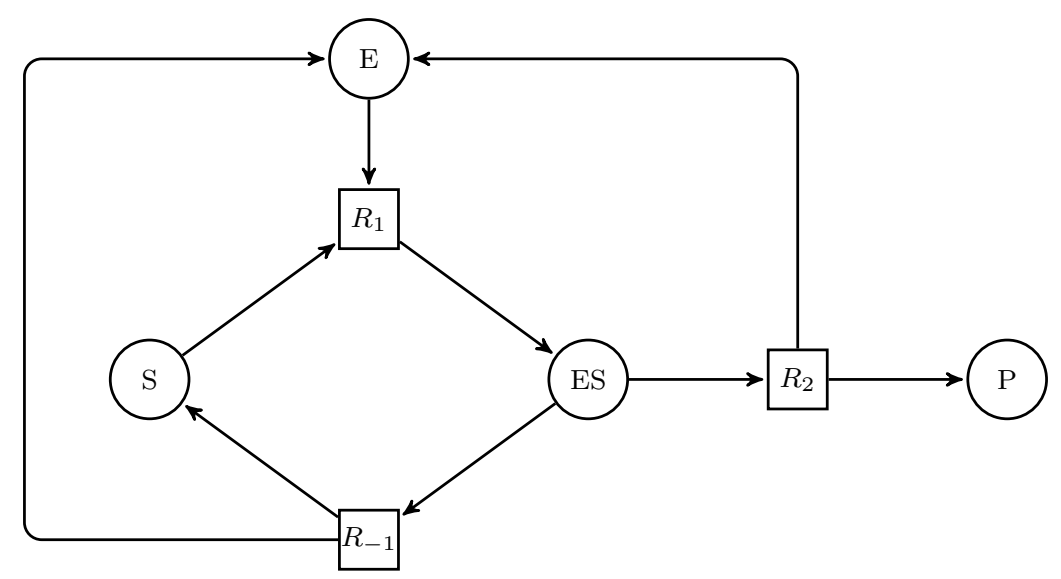

Fig. 2 Bipartite graph representation of the enzymatic reaction: $S+E \Leftrightarrow E S \Rightarrow E+P$. Arcs being all labelled with 1 , this label is not shown.

Using the same bipartite vertices but different arcs and labels, it is possible to represent the Directed Species-Reaction (DSR) graph of [15]. The arcs are now defined and labelled as follows:

$$
\begin{aligned}
\lambda\left(S_{i}, R_{j}\right) & =\frac{\partial v_{j}}{\partial x_{i}} \\
\lambda\left(R_{j}, S_{i}\right) & =y_{i j}^{\prime}-y_{i j}
\end{aligned}
$$

Note that this is not the DSR graph defined by [3] since the arcs are always directed and the labels different.

If the label $\lambda$ is zero, then there is no arc. $\lambda$ is extended to paths (resp. subgraphs) as the product of the labels of all arcs in the path (resp. subgraph). For a path $P$, we shall write $\lambda_{S R}(P)$ (resp. $\left.\lambda_{R S}(P)\right)$ for the product of labels considering only species to reaction (resp. reaction to species) arcs.

Intuitively, the $\lambda_{S R}$ represent the contribution of species to each reaction rate, whereas the $\lambda_{R S}$ describe the stoichiometric effect of reactions on each species.

Fig. 3 shows the DSR graph for the same chemical reaction network as Fig. 2.

Definition 1 A Species Hamiltonian Hooping of the DSR graph is a collection of cycles covering each of the species nodes exactly once.

The set of all species hamiltonian hoopings will be denoted by $\mathcal{H}$.

Lemma 6.2 of [15] gives a decomposition of the Jacobian in terms of the set $\mathcal{H}$ :

$$
\operatorname{det}(J)=\sum_{H \in \mathcal{H}} \sigma(H) \lambda(H)
$$

where $\sigma$ is the sign of the species hamiltonian hooping, defined as usual. Note that, as explained in [15], this is a finer decomposition than that on the hamiltonian hoopings of the classical 


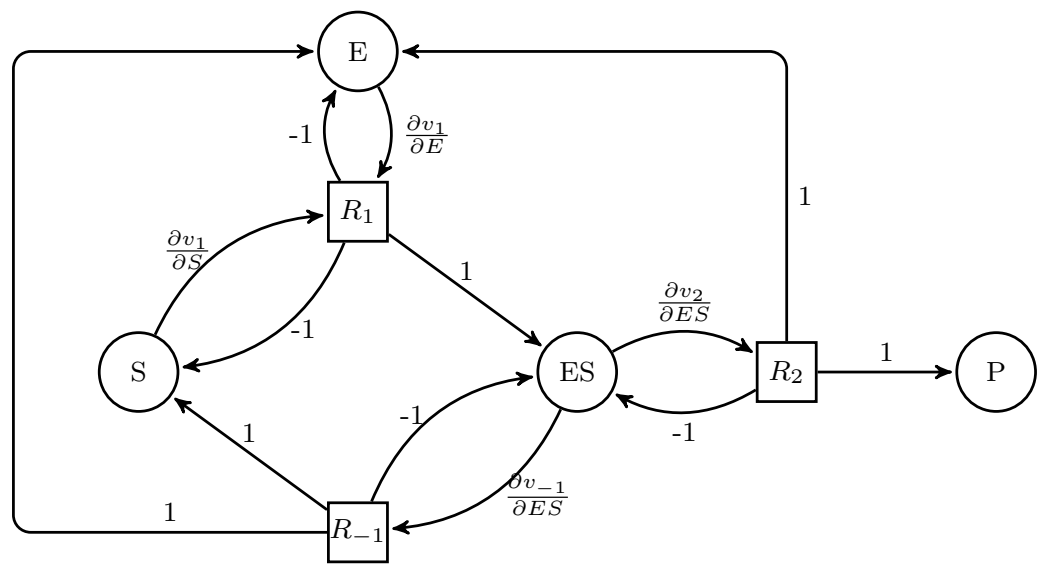

Fig. 3 DSR graph of the same enzymatic reaction: $S+E \Leftrightarrow E S \Rightarrow E+P$.

influence graph, since several species-to-species paths of length two in the DSR graph might correspond to a single arc in the influence graph.

Since the DSR graph can of course be restricted to only certain species, the same lemma can be used for any principal minor of the original determinant.

Now thanks to the fact that $\lambda(H)=\lambda_{S R}(H) \lambda_{R S}(H)$, Kaltenbach [15] groups all species hamiltonian hoopings having the same species-to-reaction arcs using an equivalence relation noted $\sim$ and obtains:

\section{Theorem $2([15])$}

$$
\operatorname{det}(J)=\sum_{[H] \in \mathcal{H} / \sim} \Lambda([H]) \lambda_{S R}(H)
$$

where $\Lambda$ is defined as:

$$
\Lambda([H])=\sum_{H^{\prime} \in[H]} \sigma\left(H^{\prime}\right) \lambda_{R S}\left(H^{\prime}\right)
$$

It is important to notice that, in its original formulation this theorem has a second part and is only stated for systems such that $\forall i, j, \partial v_{i} / \partial x_{j} \geq 0$, i.e., the $\lambda_{S R}$ are always non-negative. These systems are called NAC (Non AutoCatalytic) by Kaltenbach, but the hypothesis does not match the stronger and more usual definition of NAC systems (see for instance [4]) that forbids the production of some species to depend on its concentration. It rather matches the definition of monotonicity $[9,10]$ and allows the restriction of the sign of an element in the above sum to the different components of $\Lambda$.

Nevertheless, since we will only use the decomposition given above and whose proof does not rely on this hypothesis, we will use the theorem in its full generality.

\section{Strengthening Thomas' conjecture}

Considering Thm. 1 and applying Thm. 2 to each sub-DSR-graph corresponding to a principal minor of $-J$, one can see that a necessary condition for multistationarity is that some term of the sum is negative. This again states the usual condition about the existence of a positive cycle in the influence graph of $J$. 
We will now examine the consequences of the decomposition of this sum more precisely. In particular, we will show that many negative terms can actually be proven to cancel out with other positive terms, leaving us with more specific negative terms (and thus positive circuits) to look for.

Definition 2 The restriction of the system to a species hooping $H$ (noted $\left.\right|_{H}$ ) is the system where reactions $\left\{R_{i} \mid i \in I\right\}$ not appearing in $H$ are omitted.

Since each species appears at most once in any hooping, there is one reaction associated to each species by following the outgoing arc of that species. Note however that the same reaction can appear several times, as associated with several different species.

This definition is naturally extended to any function or graph defined by the biochemical system.

As noticed by Kaltenbach in the proof of one of the lemmas preceding Thm. 2, $\Lambda$ can be computed directly from the stoichiometric matrix $Y^{\prime}-Y$ of the biochemical system. This observation is useful in proving our first lemma:

Lemma 1 Let $H$ be a species hamiltonian hooping, if $\left.\left(Y^{\prime}-Y\right)\right|_{H}$ is not of full rank, then $\Lambda([H])=0$.

Proof As per Lemma 6.6 of $[15]$ we have $\Lambda([H])$ proportional to $\operatorname{det}\left(\left.\left(Y^{\prime}-Y\right)\right|_{H}\right)$, now, if that matrix is not of full rank, its determinant is 0 .

Remark that one can augment the usual labelling of the influence graph to contain not only the sign but also the reaction used for each arc. There is thus an arc in this reaction-labelled influence graph for each species-to-species path of length two in the DSR graph. This leads to a one-to-one correspondence between hamiltonian hoopings of the reaction-labelled influence graph and species hamiltonian hoopings of the DSR graph. Fig. 4 demonstrates this on the same example as Fig. 2.

In order to compute the signs of the arcs in this reaction-labelled influence graph, one now needs the sign of $\partial v_{i} / \partial x_{j}$ instead of that of $\partial f_{i} / \partial x_{j}$. However, even without precise kinetic values, this can be easily computed for most commonly used kinetics — see for instance [9] for the cases of mass action, Michaelis-Menten and Hill kinetics.

This allows us to state our main results:

Theorem 3 Let $F$ be any differentiable map from $\Omega$ to $\mathbb{R}^{n}$ corresponding to a biochemical reaction system. If $\Omega$ is open and $F$ has two nondegenerate zeroes in $\Omega$ then there exists some a in $\Omega$ such that:

1. The reaction-labelled influence graph $G$ of $F$ at point a contains a positive circuit $C$;

2. There exists a hooping $H$ in $G$, such that $C$ is subcycle of $H$ with $\left.\left(Y^{\prime}-Y\right)\right|_{H}$ of full rank.

Proof Because we share the same hypotheses, we can use Thm. 1 to obtain $a$ and the corresponding negative principal minor. It is then possible to apply Thm. 2 to decompose that minor according to the DSR graph as a sum of terms for each species hamiltonian hooping equivalence class, and this sum must contain a negative term.

Since only positive circuits will lead to negative terms in the usual determinant decomposition, we can now prove the proposition ab absurdum. If all negative terms appear only in some $\Lambda([H])$ such that the restriction of the stoichiometric matrix to $H$ is not of full rank, they will be cancelled out by other terms, following Lemma 1. This would lead to a contradiction. 


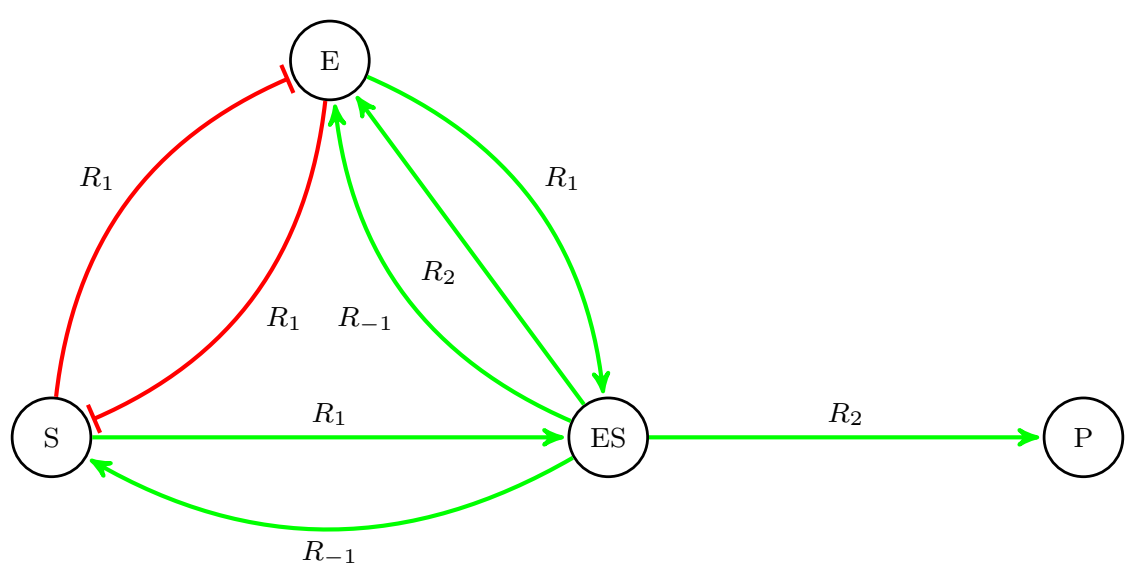

Fig. 4 Influence graph of $S+E \Leftrightarrow E S \Rightarrow E+P$. Arcs are labelled by their sign, as usual, but also by the unique reaction involved in order to obtain precisely the same species-species paths as in Fig. 3. Note for instance that there are two positive arcs from ES to E. Negative self-loops are omitted for clarity as in Fig. 1.

Note that these properties can be checked directly on the reaction-labelled influence graph and the stoichiometry matrix.

Remark that $H$ might not be hamiltonian since it is hamiltonian in a subgraph corresponding to the principal minor that is negative. See the end of Section 6 for cases when the number of species in $H$ can be known beforehand.

Let us now apply this theorem to some common cases.

Corollary 1 A necessary condition for the multistationarity of a biochemical reaction system is that there exists a positive cycle in its influence graph, using at most once each reaction.

Proof This is a direct consequence of Thm. 3 since a matrix with two identical rows is clearly singular.

It is immediate to check that the mutual inhibition resulting from bimolecular reactions-like that between $\mathrm{E}$ and $\mathrm{S}$ in our running example or between A and B in Fig. 1 - cannot fulfill these necessary conditions, since the same reaction $-R_{1}$ in Fig. $4-$ will be repeated twice.

Corollary 2 A necessary condition for the multistationarity of a biochemical reaction system is that there exists a positive cycle in its influence graph, not using both forward and backward directions of any reversible reaction.

Proof This is once again an immediate consequence of Thm. 3 since a matrix with two opposite rows is clearly singular.

One can thus remark that the mutual activation resulting from reversible reactions-like that between ES and $\mathrm{S}$ through $R_{1}$ and $R_{-1}$ in our running example or between $\mathrm{D}$ and $\mathrm{E}$ in Fig. 1-cannot fulfill these necessary conditions.

Note that this corollary corresponds to Lemma 6.9 from [15] but with a much simpler proof involving no rewiring of the influence graph.

Another information that can be extracted from the stoichiometry is the (structural) conservation laws, i.e., P-invariants of the underlying Petri net, or more simply the left kernel of the stoichiometry matrix. Finding all the conservation laws of a biochemical model might be computationally expensive, though in practice that does not seem to be the case [23], but checking 
if a given set of species are such that their sum is constant is trivial. Based on this observation one can state the following other corollary:

Corollary 3 A necessary condition for the multistationarity of a biochemical reaction system is that there exists a positive cycle in its influence graph, not using all species involved in a conservation law.

Proof This is once again an immediate consequence of Thm. 3 since a weighted sum of the columns corresponding to the conservation law is zero. Therefore a matrix containing those columns, even if some rows are forgotten via the selection of some principal minor, will be singular.

One can thus remark that the mutual activation between E and ES, that form a conservation law, through $R_{1}$ and $R_{2}$ in our running example cannot fulfill the necessary conditions for multistationarity.

The three Corollaries 1,2 and 3 do rule out most of the obvious cases that used to make Thomas' condition trivially satisfied. In particular, they show that none of the positive circuits of Figure 4 fulfill our stricter condition.

\section{Going further}

Let us now consider a two-step version of our running example, as is often considered in MAPK cascades and similar signaling pathways.

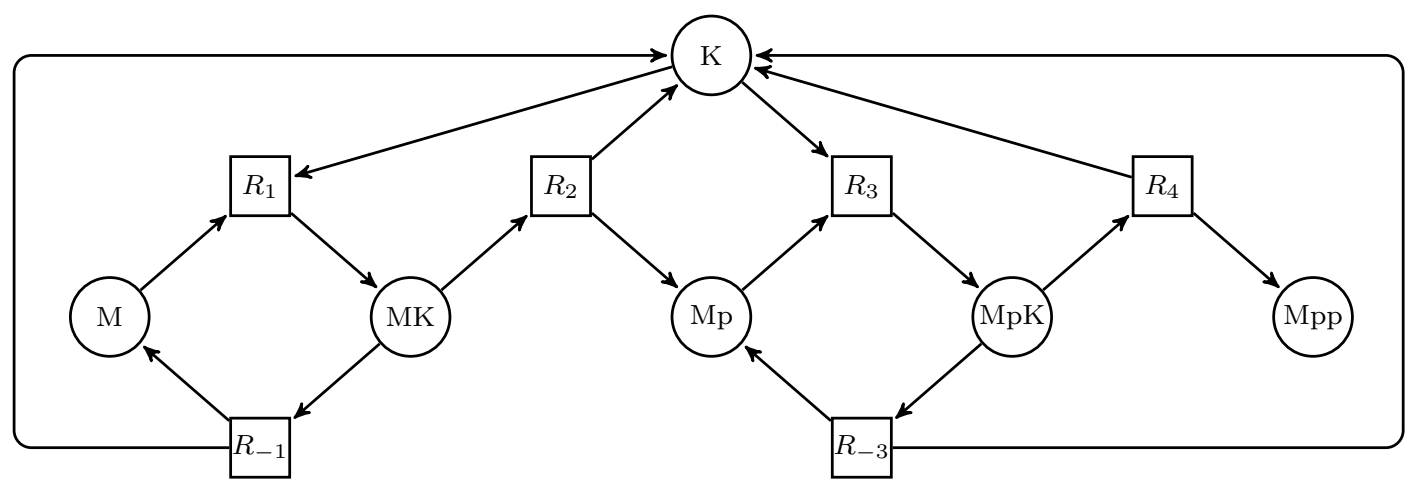

Fig. 5 Bipartite graph representation of the reactions $M+K \Leftrightarrow M K \Rightarrow K+M p \Leftrightarrow M p K=>K+M p p$. Arcs being all labelled with 1 , this label is not shown.

In this example, as before, most positive circuits can be proven not to be enough to make multistationarity possible, some come from reversible reactions like $R_{1}$ and $R_{-1}$. Some are mutual inhibitions from a single reaction like $R_{3}$, some do contain a conservation law, like the big circuit of all forms of the kinase $\mathrm{K}$ using $R_{1}, R_{2}, R_{3}$ and $R_{4}$.

However, some circuits cannot be ruled out that easily. For instance the mutual activation of $\mathrm{K}$ and MK through $R_{1}$ and $R_{2}$ is now acceptable. Indeed, in the principal minor where only these two species and Mp are considered, the hamiltonian hooping built from the said circuit and the self negative loop on Mp satisfies the hypotheses of Theorem 3.

We present here two ways to rule out this case too by transforming the system without changing the number of steady states, as noted by Soulé in [24]. 
5.1 Changing the sign of some $f_{i}$

First, one can multiply by -1 some of the $f_{i}$ without affecting the number of steady states of the system. Let us denote by $I$ the subset of $1, \ldots, n$ containing such indices.

This corresponds to a transformation of our reaction system where for each species of $I$, we exchange its stoichiometry as reactant and as product in each reaction, without modifying the rate of the reactions. In mathematical terms, for all $i$ in $I$ we exchange all $y_{i j}$ and $y_{i j}^{\prime}$, but all $v_{j}$ remain untouched. This is possible since in Theorem 2 we took care not to use any hypothesis on the $v_{j}$.

The resulting labelled reaction graph is the same as before but with the color (i.e., sign) of the arcs ending in species of $I$ reversed. Any such graph should actually fulfill our conditions in order for the original system to be able to produce multistationarity.

Corollary 4 A necessary condition for the multistationarity of a biochemical reaction system is that there exist positive cycles fulfilling condition 2 of Theorem 3 in the influence graph corresponding to its Jacobian, and in any graph obtained from it choosing a set of species and by reversing the sign of all arcs that have as target some species belonging to that set.

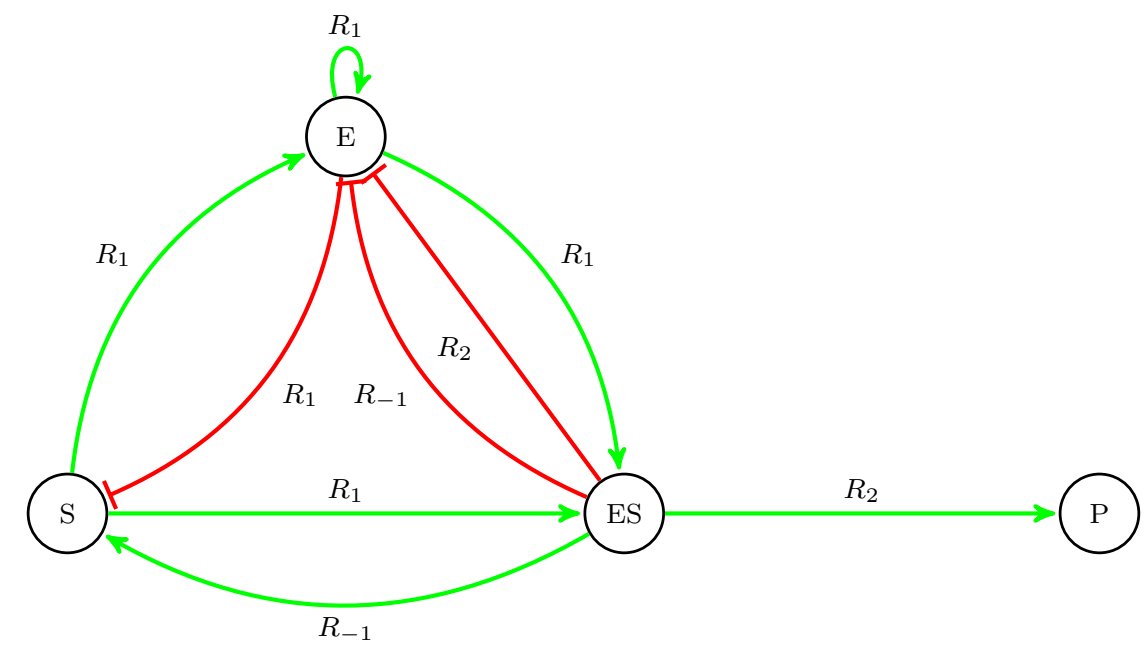

Fig. 6 Influence graph of Fig. 4 but with arcs ending in $\{E\}$ inversed. Note that the self-loop on $E$ has become positive. All other positive cycles contain either twice $R_{1}$ or both $R_{1}$ and $R_{-1}$.

Figure 6 shows the result of choosing $I=\{E\}$ in our initial example. It should be noted that there is now a positive loop on $\mathrm{E}$ since it had a negative loop beforehand. When trying to prove that a system cannot exhibit multistationarity, it might thus be worth restricting the search for an $I$ to sets that contain at least some of the vertices belonging to the positive circuit that satisfies our hypotheses in the initial graph, and to sets that do not contain any vertex that had a negative self-loop in the initial graph.

In the new example, since both K and MK have negative self-loops, Corollary 4 cannot rule out the circuit. 


\subsection{Permuting the indices of some $f_{i}$}

Another transformation of the system that does not change its number of steady states is to multiply the Jacobian by a permutation, i.e., to permute the indices of some $f_{i}$.

In biochemical system terms, this can be done by applying the permutation to the species appearing in the reactions (as products or rectants), and thus to the $y_{i j}$ and $y_{i j}^{\prime}$ but not to the rates $v_{j}$.

This transforms only the species-to-reaction arcs of the DSR graph, rewiring them according to the permutation. In the labelled influence graph, the signs and reaction labels do not change, but the arcs are rewired such that their target corresponds to the image by the permutation. All such graphs should once again fulfill our conditions in order for the original system to be able to exhibit multistationarity.

Corollary 5 A necessary condition for the multistationarity of a biochemical reaction system is that there exist positive cycles fulfilling condition 2 of Theorem 3 in the influence graph corresponding to its Jacobian, and in any graph obtained from it choosing a permutation of the species and by rewiring the arcs' target according to the permutation.

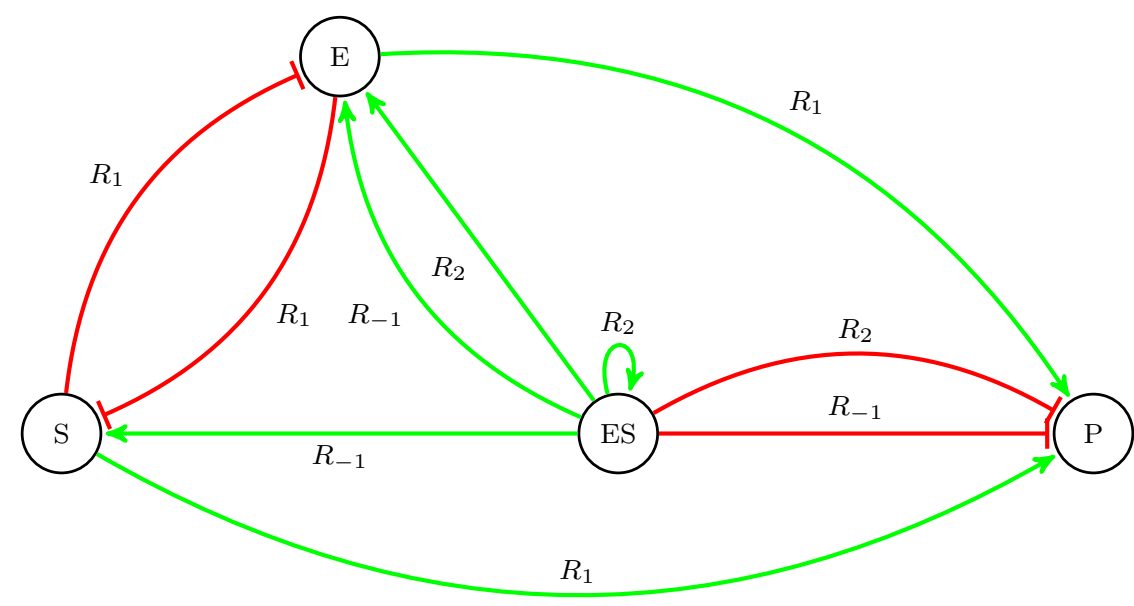

Fig. 7 Influence graph of Fig. 4 but with arcs ending in P and ES substituted. Resulting negative self loops are, as usual, not shown, but note that the negative arcs from ES to P corresponds to negative self loops on ES in the original graph.

Figure 7 shows the result of choosing a permutation of ES and P in our initial example. It should be noted that there is now a positive loop on ES since there was a positive arc from it to $\mathrm{P}$ beforehand. When trying to prove that a system cannot exhibit multistationarity, it might thus be worth restricting the search for a permutation to those that do not map a vertex to any other vertex such that the first one has a positive arc to it.

Actually, as noted in [24] the two above corollaries can be combined.

Figure 8 shows that by choosing the permutation of $\mathrm{K}$ and Mpp in our new example, and by changing the sign on $\mathrm{K}$ there is no more positive cycle belonging to a hooping of full rank. This proves that the system cannot exhibit multistationarity. 


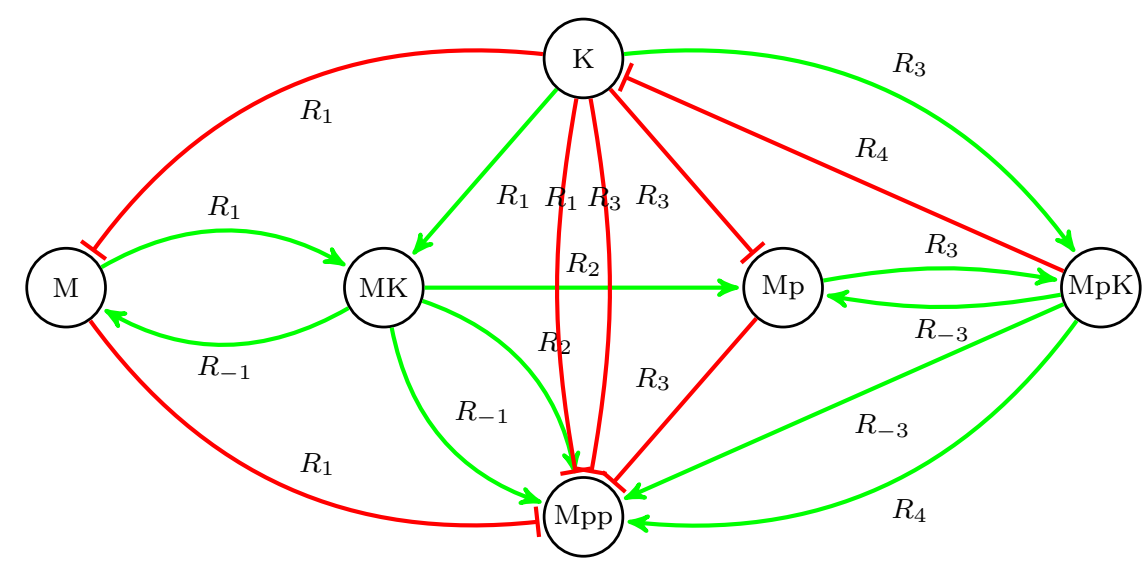

Fig. 8 Influence graph of Fig. 5 but with arcs ending in $\mathrm{K}$ and Mpp substituted, and then the only arc ending in $\mathrm{K}$ changed of sign. Resulting negative self loops are, as usual, not shown, but note that the negative arcs from $\mathrm{K}$ to Mpp corresponds to the negative self loops on $\mathrm{K}$ in the original graph. All positive circuits remaining can be ruled out by one of our corollaries as being a possible source of multistationarity.

\section{Related work}

The results we present in this paper bear some similarity with many existing works from the Chemical Reaction Network Theory community. In particular the works of Banaji [2] and Craciun [6] that tackle multistationarity. Note however that the methods are different (even if another homonymous DSR graph is used) and particularly the hypotheses on the biochemical networks handled. Even with the recent works [11] lifting the requirement for having outflows for each species, the results usually require to have only mass-action kinetics, or at least monotone rates and no auto-catalysis (i.e. NAC kinetics, see also the discussion after Theorem 2). Note that this kind of restriction forbids all the graph transformations of Section 5. Moreover, the conclusions are different: while the methods we present here do not handle all the examples of [8], conversely their method does not provide any conclusion for our latest MAPK example, since the c-pair $\mathrm{K}$ Mp from $R_{2}$ is split between the two even-cycles $R_{1}, R_{2}$ and $R_{2}, R_{3}$. The approaches are thus complementary, even when restricting the results of the current paper to specific types of kinetic rates.

There has been an important amount of work from Angeli and Sontag about graphical requirements in chemical reaction networks. However as they state themselves in [1] about the work of Craciun and Feinberg, "While we present global stability results, that work is concerned with the problem of identifying which chemical reaction networks may have multiple equilibria in their stoichiometry classes." Indeed, they characterize monotonicity, persistence and convergence properties of systems. These different properties in turn rely on different hypotheses than ours: they require once again NAC kinetics, whereas the present article does not.

Finally, a result that might appear similar to Theorem 3 but restricted to mass-action systems is the second Theorem of Mincheva and Roussel [17]. Note however that their result is about the order, i.e., number of species, of the hamiltonian hooping, implying that when their hypotheses are satisfied, the $H$ we look for in Theorem 3 covers $r$ species, where $r$ is the rank of the Jacobian. Their result also requires the explicit computation of $K_{S_{r}}$, the coefficient (similar to our $\Lambda$ ) of their critical fragment (similar to our equivalence classes over hoopings). In contrast, we provide graphical conditions for which we know from the stoichiometry - and without any hypothesis on the kinetics - that this coefficient will actually be 0 , and thus that some other positive cycle 
should be found. Finally, once again, because of the restrictions put on the kinetic rates, their approach cannot benefit from the graph transformations presented in Section 5.

\section{Conclusion}

Using the structural information, notably from the stoichiometric matrix, of a biochemical system, we have been able to state a more strict version of the famous Thomas' necessary condition for multistationarity.

Of course, since we have made no hypothesis on the kinetic rate functions, one can easily represent any dynamical system as such biochemical reactions. Typically one would then have one reaction for each variable with rate corresponding to its derivative. In this case the supplementary hypothesis of our theorems collapse, leaving us with the usual conditions. However for more usual biochemical systems it brings a concrete difference, as illustrated by Cor. 1, 2 or 3, and if necessary their application to many transforms of the influence graph as seen in Corollaries 4 and 5 .

In particular, the obvious cases where Thomas' condition was trivially satisfied, as illustrated by Fig. 1 and Fig. 4, can now easily be ruled out.

The results are local (i.e., the cycle does exist for some $a$ in the phase space) since they rely on local theorems as was the case in [24]. Since the other Thomas' condition, on oscillations, is not [21] one cannot directly apply the same reasoning we used to Hurwitz Determinants. It might still be worth investigating, for instance when the signs of the influences in the labelled influence graph are known to be constant.

The same locality argument makes it difficult to interpret our stronger condition as some version of Thomas' circuit functionality for the continuous case. It is rather related and complementary to the whole domain of the structural analysis of (bio)chemical reaction systems, as pioneered by Chemical Reaction Network Theory. As such, we believe that our stronger necessary condition is enough to make circuit analysis a more worthwhile tool in the arsenal of the structural analyst of biochemical systems.

Acknowledgements We would like to thank Steven Gay, a Ph.D. student in our team, who was the first to formulate the conjecture proven in this article, as well as its variant on oscillations. We also thank Christophe Soulé for very interesting discussions that have led to Section 5. Finally we thank the whole Contraintes team for numerous fruitful discussions on this topic.

\section{References}

1. David Angeli, Patrick De Leenheer, and Eduardo Sontag. Graph-theoretic characterizations of monotonicity of chemical networks in reaction coordinates. Journal of Mathematical Biology, 61(4):581-616, 2010.

2. Murad Banaji. Cycle structure in sr and dsr graphs: implications for multiple equilibria and stable oscillation in chemical reaction networks. In Transactions on Petri Nets and Other Models of Concurrency V, pages 1-21. Springer-Verlag, 2012.

3. Murad Banaji and Gheorghe Craciun. Graph-theoretic approaches to injectivity and multiple equilibria in systems of interacting elements. Communications in Mathematical Sciences, 7(4):867-900, 2009.

4. Murad Banaji, Pete Donnell, and Stephen Baigent. P matrix properties, injectivity, and stability in chemical reaction systems. SIAM Journal on Applied Mathematics, 67(6):1523-1547, 2007.

5. Oliver Cinquin and Jacques Demongeot. Positive and negative feedback: striking a balance between necessary antagonists. Journal of Theoretical Biology, 216(2):229-241, 2002.

6. Gheorghe Craciun and Martin Feinberg. Multiple equilibria in complex chemical reaction networks: I. the injectivity property. SIAM Journal on Applied Mathematics, 65(5):1526-1546, 2005.

7. Gheorghe Craciun and Martin Feinberg. Multiple equilibria in complex chemical reaction networks: Ii. the species-reaction graph. SIAM Journal on Applied Mathematics, 66(4):1321-1338, 2006.

8. Gheorghe Craciun, Yangzhong Tang, and Martin Feinberg. Understanding bistability in complex enzymedriven reaction networks. PNAS, 103(23):8697-8702, 2006. 
9. François Fages and Sylvain Soliman. Abstract interpretation and types for systems biology. Theoretical Computer Science, 403(1):52-70, 2008.

10. François Fages and Sylvain Soliman. From reaction models to influence graphs and back: a theorem. In Proceedings of Formal Methods in Systems Biology FMSB'08, number 5054 in Lecture Notes in Computer Science. Springer-Verlag, February 2008.

11. Elisenda Feliu and Carsten Wiuf. Preclusion of switch behavior in reaction networks with mass-action kinetics. Applied Mathematics and Computation, 219(4):1449-1467, 2012.

12. David Gale and Hukukane Nikaido. The jacobian matrix and global univalence of mappings. Mathematische Annalen, 159:81-93, 1965.

13. Jean-Luc Gouzé. Positive and negative circuits in dynamical systems. Journal of Biological Systems, 6:11-15, 1998.

14. Michael Hucka, Stefan Hoops, Sarah M. Keating, Le Novère Nicolas, Sven Sahle, and Darren Wilkinson. Systems biology markup language (SBML) level 2: Structures and facilities for model definitions. Nature Precedings, December 2008.

15. Hans-Michael Kaltenbach. A unified view on bipartite species-reaction and interaction graphs for chemical reaction networks. arXiv, October 2012.

16. Marcell Kaufman, Christophe Soulé, and René Thomas. A new necessary condition on interaction graphs for multistationarity. Journal of Theoretical Biology, 248:675-685, 2007.

17. Maya Mincheva and Marc R Roussel. Graph-theoretic methods for the analysis of chemical and biochemical networks. i. multistability and oscillations in ordinary differential equation models. Journal of mathematical biology, 55(1):61-86, 2007.

18. Erik Plahte, Thomas Mestl, and Stig W. Omholt. Feedback loops, stability and multistationarity in dynamical systems. Journal of Biological Systems, 3(02):409-413, 1995.

19. Elisabeth Remy, Paul Ruet, and Denis Thieffry. Graphic requirements for multistability and attractive cycles in a boolean dynamical framework. Advances in Applied Mathematics, 41(3):335-350, 2008.

20. Adrien Richard and Jean-Paul Comet. Necessary conditions for multistationarity in discrete dynamical systems. Discrete and Applied Mathematics, 155:2403-2413, 2007.

21. Adrien Richard and Jean-Paul Comet. Stable periodicity and negative circuits in differential systems. Journal of Mathematical Biology, 63(3):593-600, 2011.

22. El Houssine Snoussi. Necessary conditions for multistationarity and stable periodicity. Journal of Biological Systems, 6:3-9, 1998.

23. Sylvain Soliman. Invariants and other structural properties of biochemical models as a constraint satisfaction problem. Algorithms for Molecular Biology, 7(15), May 2012.

24. Christophe Soulé. Graphic requirements for multistationarity. ComplexUs, 1:123-133, 2003.

25. René Thomas. On the relation between the logical structure of systems and their ability to generate multiple steady states or sustained oscillations. Springer Ser. Synergetics, 9:180-193, 1981. 